\title{
3D Arrayed Microwave Tomographic System for Medical Imaging
}

\author{
M. Guardiola ${ }^{(1)}$, L. Jofre*(1), F. Gedda ${ }^{(2)}$, S. Capdevila ${ }^{(1)}$, J. Romeu $^{(1)}$ and S. Blanch $^{(1)}$ \\ (1) AntennaLab, UPC, C/ Jordi Girona 3-4, 08034 Barcelona (Spain) \\ (2) Politecnico di Torino, Corso Duca degli Abruzzi 24, 10129 Torino (Italy) \\ E-mail: \{marta.guardiola, scapdevila, jofre\}@.tsc.upc.edu
}

\section{Introduction}

Microwaves and in particular the ultra wideband (UWB) frequency range has been exploited for the last years due to its potential in medical imaging research field. Microwave imaging has been proposed as a complementary or alternative scanning method to X-ray imaging, ultrasounds or MRI, being the more established imaging tools however presenting limitations [1]. The potential of microwave imaging for practical clinical use is significant due to a wide variety of advantages, which satisfy the requirements for an efficient imaging system, including the use of low-power non-ionizing radiation, low-cost system implementation, detection of malignancy at early stage and patient comfort. Moreover the tradeoff between penetration and resolution provided by UWB frequencies, together with its capability to experiment highly sensitive and specific interactions depending on the tissue nature, drove its application to the detection of breast tumors or even brain strokes.

Active microwave imaging techniques are generally categorized as tomographic and radar-based techniques. The design and fabrication of antennas and systems for each application present particular requirements. Typical antenna design examples include monopoles, slots, bowties, waveguides or horn antennas [2], [3], [4]. While in time domain radar methods a non-resonant antenna is needed to avoid the pulse degradation; in tomographic case, the main issue is to collect a sufficient number of scattered field points to recover the currents in the inverse scattering problem. To do that, for a given transmitter, the field is measured at all the receiving antennas and this procedure is successively repeated for each transmitter. This can be accomplished by either using a real array of multiplexed antennas or virtually creating it with the movement of two antennas. While the second approach avoids the coupling between antennas and the complexity of the multiplexing system; it is not suitable for a real time system due to the prolonged duration of the measurement. Hence most of the times the virtual array approach is used for the early stages of the prototype converging into antenna arrays for the clinical prototype.

In this paper, a combined tomographic imaging system comprising the rotation of a multiplexed linear antenna array around the object under test is designed. With this system a cylindrical sampling is obtained, allowing to scan realistic phantoms without important restrictions on its shape. This, combined with the non-application specific 3D UWB Hybrid Focusing reconstruction algorithm, allows to use this system in a wide range of applications. The design of a multiplexed array of UWB antennas for medical applications entails significant challenges including a large impedance bandwidth, small size and low coupling between elements, which will be treated in this paper.

\section{Reconstruction Algorithm}

The reconstruction algorithm imposes additional requirements to the antenna array. In particular for a cylindrical imaging system composed by $N_{V}$ rings of $N_{H}$ antennas of radius $a$ separated a distance $s$ in the vertical direction, $N_{H}$ has to be chosen in order to avoid loss of information according to (see Fig. 1):

$$
N_{H} \geq \frac{4 \pi a f \sqrt{\epsilon_{0}}}{c_{0}}
$$

In the same fashion, $s$ must be smaller than half a wavelength for the whole operating band in order to fulfill Nyquist sampling criteria. 


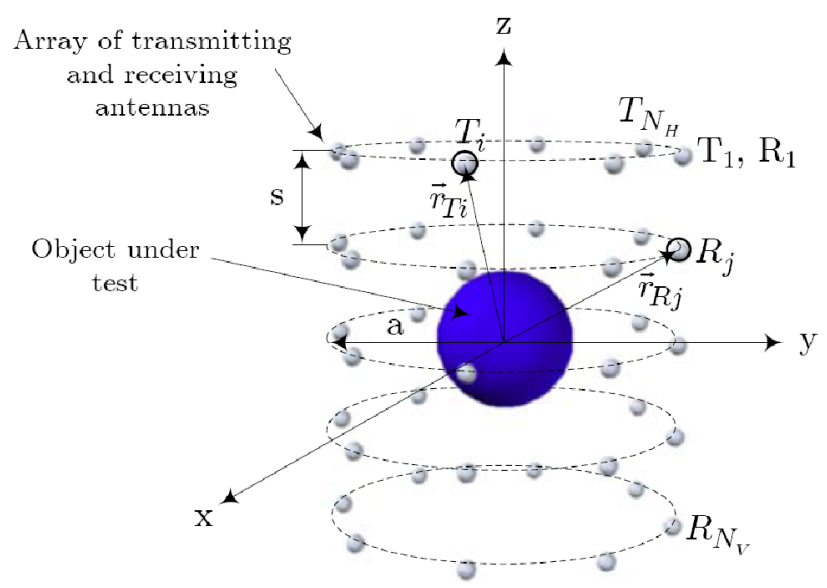

Fig. 1. Sketch of the cylindrical antenna

Under this sampling conditions, the 3D UWB Hybrid Focusing algorithm [5] can be used to recover an image of the contrast profile $(C(\vec{r}))$ which is related to the complex permittivity of the object under test $(\epsilon(\vec{r}))$ and the external medium $\left(\epsilon_{0}\right)$ :

$$
C(\vec{r})=1-\frac{\epsilon(\vec{r})}{\epsilon_{0}}
$$

The contrast profile is obtained by the incoherent multi-frequency combination of coherent multi-view focused images calculated by applying the subsequent equation:

$$
\tilde{C}\left(\vec{k}_{1}+\vec{k}_{2}\right)=\frac{1}{j \omega \epsilon_{0}} \sum_{i=1}^{N_{H} N_{V}} \sum_{j=1}^{N_{H} N_{V}} E^{s}\left(\vec{r}_{T_{i}} ; \vec{r}_{R_{j}}\right) I\left(\vec{r}_{T_{i}} ; \vec{k}_{1}\right) I\left(\vec{r}_{R_{j}} ; \vec{k}_{2}\right)
$$

Where the spectrum of the contrast profile $(\tilde{C}(\vec{k}))$ is expressed as the combination of the scattered field measurements at all the cylindrical antenna positions $\left(E^{s}\right)$ weighted by the current distribution coefficients $(I)$ that applied on our cylindrical antenna would obtain a plane wave towards $\vec{k}_{i}$. Fig. 2 show the results of the reconstruction of a simulated simplified model of brain presenting a spherical blood vessel inside (see Fig. 2a for a sketch of the geometry and Table I for the permittivity values used in the simulation of the brain model). Fig. $2 b$ represents the intersection of the three main planes intersecting at the center of the vessel and Fig. $2 \mathrm{c}$ is a detail of the horizontal plane. This results show the good performance of the algorithm for retrieving the shape and the different layers and also for detecting the blood vessel.

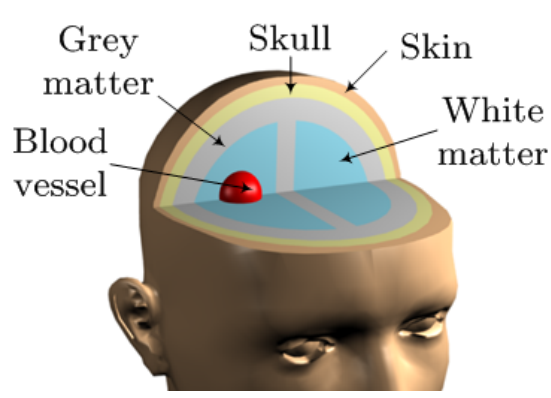

(a) Simulated model.

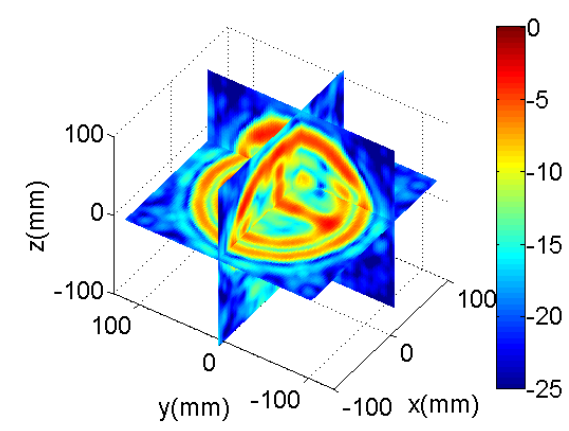

(b) Intersection of the 3 main planes.

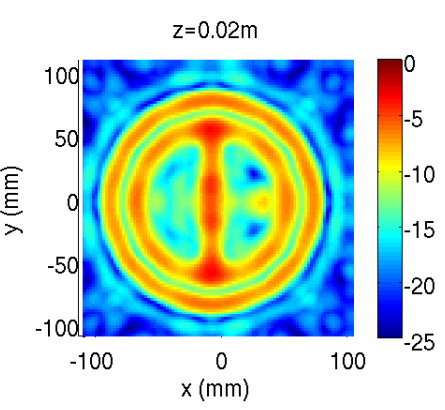

(c) Horizontal plane.

Fig. 2. Reconstruction of a simulated simplified model of brain with a blood vessel $\left(N_{V}=25, N_{H}=32\right.$, $a=150 \mathrm{~mm}$ and $s=10 \mathrm{~mm}$ ). 


\begin{tabular}{c|c|c|c|c|c|c}
\hline Tissue & Exterior & Skin & Skull & Grey matter & White matter & Blood vessel \\
\hline $\boldsymbol{\epsilon}_{\boldsymbol{r}}$ & 40 & 40 & 13 & 50 & 40 & 50 \\
\hline
\end{tabular}

Table I. Dielectric properties of the simplified brain model.

\section{Array Design}

The implementation of the cylindrical imaging system will be done as depicted in Fig. 3a. The object under test and the receiving array are mounted on two independent high-resolution rotary stages around the same rotation axis, while the transmitting array remains in a fixed location. Both transmitting and receiving arrays are composed by $N_{V}$ elements which can be selected as the active element by means of a SP8T (Single Pole 8 Throw) governed by a microcontroller. The combined rotation of the object and the receiving array together with a convenient multiplexing sequence, allows to construct two virtual concentric cylindrical arrays for the transmitter and receiver respectively. The received signal is measured using a 2-port VNA which performs a frequency sweep in the UWB frequency range $(3.1-10.6 \mathrm{GHz})$. By means of a computer the whole measurement process (switching, rotation sequence and data acquisition) is automated and the results stored and processed.

A microstrip bow-tie slot antenna has been chosen as the radiating element for its easy manufacturing and feeding together with its capability to produce large bandwidths in a compact size, see Fig. 3b. It consists of a sandwiched structure with two metallic grounded substrates separated by a light foam layer. The grounded substrate close to the target contains the radiating bow-tie slot in the exterior conductor and a microstrip feeding line in the interior side. The second grounded plane is situated at a distance of a quarter-wavelength at the center frequency from the radiating structure and acts as a reflector in order to produce a unidirectional radiation towards the volume under investigation. Rogers $\mathrm{RO} 4003 \mathrm{C}\left(\epsilon_{\mathrm{RO} 4003}=\right.$ 3.4 ) of $0.8 \mathrm{~mm}$ in thickness has been chosen as substrate for its good stability in frequency. The antenna is fed by a tappered $50 \Omega$ microstrip line short circuited with the slot ground plane.

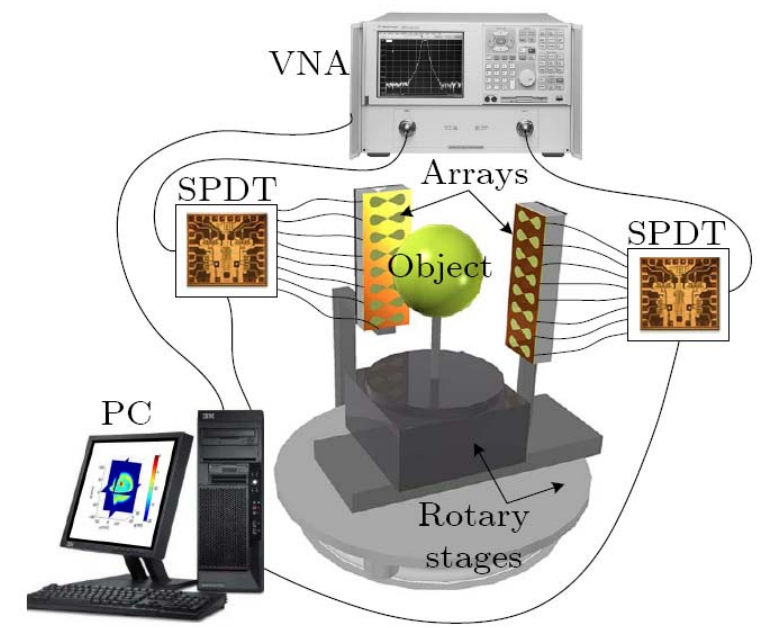

Fig. 3a. System schematic.

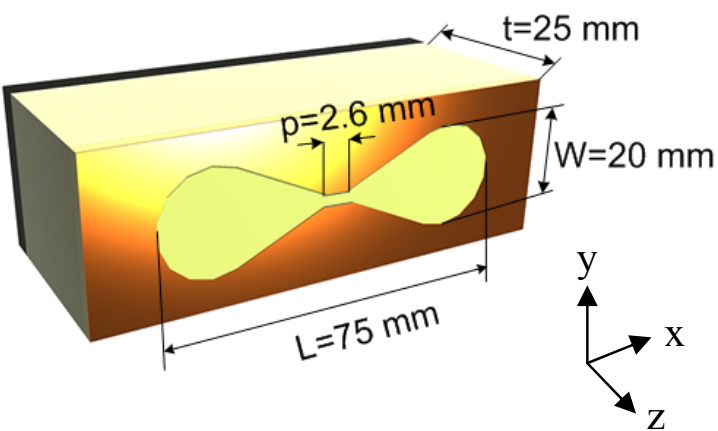

Fig. 3b. Geometry of the single element.

\section{Results}

Fig. 4a shows the results of a HFSS simulation of the single element in terms of input match. As can be seen, a good matching is accomplished between 3 and $12 \mathrm{GHz}$ representing a bandwidth of 1:4. Fig. $4 \mathrm{~b}$ includes the radiation patterns corresponding to $\mathrm{XZ}$ and $\mathrm{YZ}$ planes for the center frequency. It can be seen that the antenna polarization is merely lineal and vertical as required for the reconstruction algorithm and 
the radiation pattern presents a maximum towards $+\hat{z}$. The radiation towards $-\hat{z}$ has been reduced thanks to the reflector. The measured results will be presented at the final presentation.

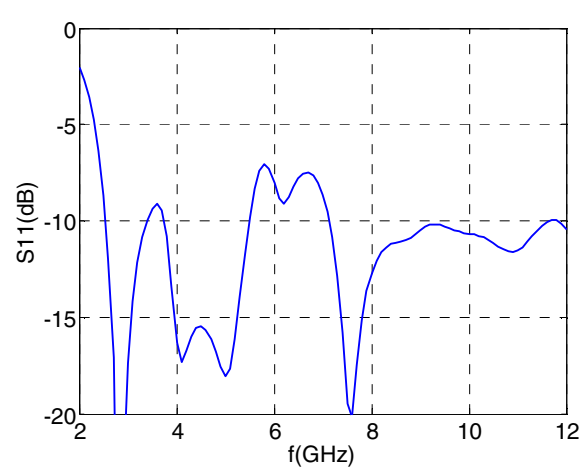

Fig. 4a. Input matching of the antenna.

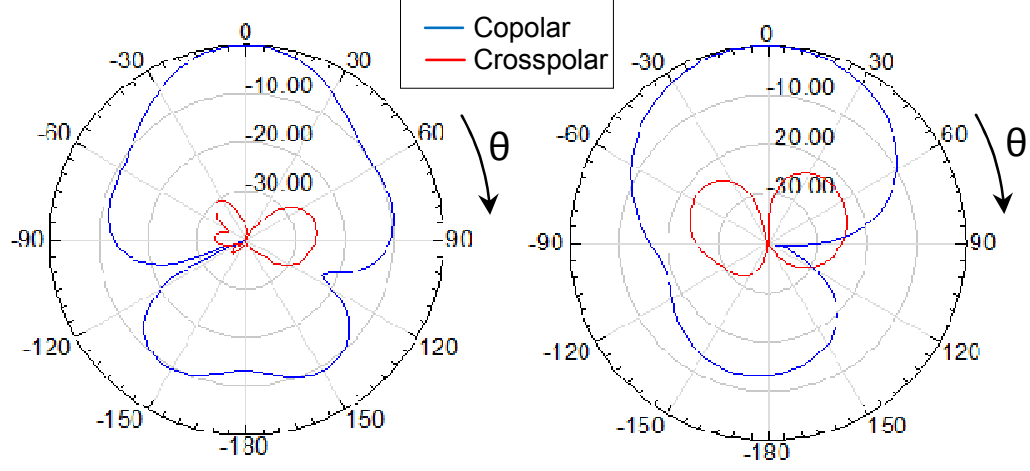

Fig. 4b. Radiation patterns. Copolar and crosspolar components for the XZ plane (left) and YZ plane (right).

\section{Conclusions}

A 3D cylindrical microwave tomographic system for medical imaging applications has been proposed. The cylindrical sampling has been accomplished rotating a multiplexed column array for the transmitting and receiving ends with the aim to reduce the acquisition time. The array has been designed to obtain a broad bandwidth in a compact size and a vertical electric field and a radiation pattern directed to the body under test.

\section{Acknowledgements}

This work was supported in part by the Spanish Interministerial Commission on Science and Technology (CICYT) under projects TEC2007-66698-C04-01/TCM and CONSOLIDER CSD2008-00068 and by Ministerio de Educación y Ciencia through the FPU fellowship program.

\section{References:}

[1] Douek, M.; Davidson, T.; Taylor, I.; "Breast Cancer Imaging - What are the Optimal Modalities?," Eur. J. Surg. Oncol., vol. 24, no. 6, pp. 573-382, 1998

[2] Hein, M.A.; Geyer, C.; Helbig, M.; Hilger, I.; Sachs, J.; Schwarz, U.; Seifert, F.; Stephan, R.; Thiel, F.; , "Antennas for ultra-wideband medical sensor systems," Antennas and Propagation, 2009. EuCAP 2009. 3rd European Conference on, pp.1868-1872, 23-27 March 2009

[3] Meaney, Paul M.; Paulsen, Keith D.; Fanning, Margaret W.; Poplack, Steven P.; Tosteson, Tor D.; Li, Dun; Yagnamurthy, Navin K. V.; , "Microwave Breast Imaging with a Non-Contacting, Monopole Antenna Array," Microwave Conference, 2000. 30th European, pp.1-4, Oct. 2000

[4] Craddock, I.J.; Klemm, M.; Leendertz, J.; Preece, A.W.; Benjamin, R.; , "An Improved Hemispeherical Antenna Array Design for Breast Imaging," Antennas and Propagation, 2007. EuCAP 2007. The Second European Conference on , pp.1-5, 11-16 Nov. 2007

[5] Guardiola, M.; Jofre, L.; Capdevila, S.; Blanch, S.; Romeu, J.; , "Toward 3D UWB Tomographic Imaging System for Breast Tumor Detection," Antennas and Propagation, 2010. EuCAP 2010. 4rd European Conference on , 12-16 April 2010 